Int J Infect Microbiol 2012;1(1):24-28

\section{Class 1 integron element in Thai Acinetobacter baumannii reveals a linkage to the European clone I}

\author{
Thapa $B,{ }^{1}$ Tribuddharat $C,{ }^{2 *}$ Srifuengfung $S,{ }^{2}$ \\ Dhiraputra $\mathrm{C}^{2}$
}

${ }^{1}$ Genesis Laboratory and Research, Kathmandu Nepal, ${ }^{2}$ Department of Microbiology, Faculty of medicine Siriraj Hospital, Mahidol University, Bangkok, Thailand

*Correspondence to: Dr. Chanwit Tribuddharat, Department of Microbiology, Faculty of medicine Siriraj Hospital, Mahidol University, Bangkok, Thailand, email: sictb@mahidol.ac.th, Tel. No.: (+066)-816326331

\begin{abstract}
BACKGROUND: Class 1 integron element is innate to most of the multidrug resistant Acinetobacter baumannii and its spread is common among international clones worldwide. The aim of this study was to document the presence of bla $a_{\mathrm{VEB}-1}$ harboring class 1 integron element and its gene cassettes in Thai $A$. baumannii in relation to $A$. baumannii European clone I, AYE strain.
\end{abstract}

MATERIALS AND METHODS: Thirty seven carbapenem resistant $A$. baumannii isolates identified in routine microbiology laboratory of Siriraj Hospital, Bangkok were studied. The dot blot hybridization was performed to detect class 1 integron element integrase gene. PCR was used to amplify bla $a_{\mathrm{VEB}-1}$, arr-2, cmlA, bla $a_{\mathrm{OXA}-10}$ resistance cassettes, and variable region of class 1 integron element. $b l a_{\mathrm{VEB}-1}$ gene was localized by southern blot hybridization.

RESULTS: The prevalence of class 1 integron element was $86.48 \%$ in the isolates studied. The bla $_{\text {VEB-1 }}$ was present in 7 isolates however the location of $b l a_{\text {VEB-1 }}$ gene was different in different isolate. Four isolates (Ab03-168, Ab04-28, Ab0820, and Ab08-22) harbored calss 1 integron element variable region sized $5.5 \mathrm{~kb}$ as described in strain AYE. However, bla $a_{\mathrm{VEB}-1}$ was only amplified from Ab03-168. The cassette organization in this isolate was $5^{\prime} \mathrm{CS}$-aadB-bla $a_{\mathrm{VEB}-1}$-arr-2-cmlA-bla ${ }_{\text {OXA- }}$ 10-aadA1-3'CS. The class 1 integron element similar to the element identified in genomic resistance island, AbaRI of European clone I, AYE was identified in Thai $A$. baumannii.

CONCLUSIONS: $b l a_{\text {VEB-1 }}$ harboring class 1 integron element with minor cassette variation was identified in Thai $A$. baumanni isolate which might suggest the spread of this resistant cassette or the spread of the European clone I in Thailand. Monitoring of the global spread of multi-resistant A. baumannii is mandatory to control the spread of resistant genes and this multi-resistant pathogen.

KEY WORDS: Class 1 integron element, intI1, bla $a_{\mathrm{VEB}-1}$, International clones, $A$. baumannii

Article submitted 20 July. Reviewed 30 August. Author correction 7 September. Final version accepted 25 September 2012 


\section{INTRODUCTION}

A. baumannii is an emerging nosocomial pathogen inflicting debilitating patients in intensive care units. The increasing resistance to most of the sophisticated antibiotics (carbapenems, tigecycline, and colistin) used in clinical practice or spread of multi-resistant international clones of $A$. baumannii is responsible for high morbidity and mortality (30$70 \%)^{1-3}$ The genetic basis of resistance to these antibiotics have been contributed by innate resistant determinants, acquisition of resistant genes, integron elements to genomic resistance islands (GRI), and mutation in drug target sites. ${ }^{4}$ Integron element carries resistant gene cassettes which is a mobile genetic element. ${ }^{5}$ Among three classes of integron elements, class 1 integron element is the commonest in Gram negative bacteria including A. baumannii.

Integron element can be considered as a minigenomic resistant island that carries single to multiple drug resistant cassettes and isolates possessing it correlates with resistant phenotype. ${ }^{1}$ Resistance gene cassettes to aminoglycoside $(a a d B$, aac3, aadA1, and aacA4), $\beta$-lactamas (bla ${ }_{\mathrm{VEB}-1,{ }^{1}}$

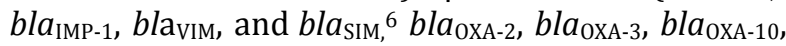
$b l a_{\text {OXA-21 }}, b l a_{\text {OXA-20, and, }}$ bla $a_{\text {OXA-37, }}, 7$ sulphonamides (dfr1), ${ }^{1}$ chloramphenicol (cmlA, cmlA5, and catB), and rifampicin $(a r r-2)^{1,8,9}$ are class 1 integron borne and have been described in international clones of A. baumannii. The largest GRI, AbaR1 has been described in French strain AYE, is $86 \mathrm{~Kb}$, and harbors three class 1 integron elements. ${ }^{1}$ The largest integron element in AbaR1 is a composite class 1 integron element consisting of bla $a_{\mathrm{VEB}-1}, a a d B$, arr-2, cmlA, bla OХA-10, and a adA1 cassettes. ${ }^{1}$

The study of class 1 integron elements and these resistance gene cassettes in Enterobacteriaceae and non-Enterobacteriaceae is common in this hospital and abroad. However, the presence of bla ${ }_{\mathrm{VEB}-1}$ harboring class 1 integron element, its cassette organization in relation to $b l a_{\mathrm{VEB}-1}$ harboring class 1 integron element of A. baumannii, European clone I, AYE strain has not been studied. Here, we studied the presence of $b l a_{\mathrm{VEB}-1}$ harboring class 1 integron element and its gene cassettes organization in Thai $A$. baumannii in relation to European clone I, $A$. baumannii AYE strain.

\section{MATERIALS AND METHODS}

Bacterial strains, DNA extraction, and controls: Thirty seven clinical isolates of carbapenem resistant-Acinetobacter baumannii (CRAB) from Sirirai Hospital, Bangkok, Thailand were studied. ${ }^{11}$
Chromosomal DNA was extracted using the Genomic DNA purification kit (Puregene, Minneapolis, Minnesota, USA) following the manufacturer's protocol. The pCTF202, a plasmid construct carrying an incomplete class 1 integron element (5'CS- bla $a_{\text {VEB-1 }}$ arr-2-cmlA-truncated bla OXA- $_{\text {OX- }}$ $\left.{ }_{10}\right)^{8}$ and $P$. aeruginosa strain, 58 carrying a complete

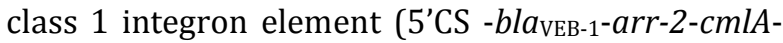

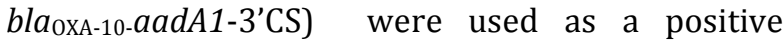
control in PCR amplification, dot blot hybridization, and cassette characterization of bla $a_{\mathrm{VEB}-1}$ containing class 1 integron element.

Polymerase chain reaction (PCR): PCR amplification was done in $50 \mu \mathrm{l}$ reaction volumes containing $0.2 \mu \mathrm{M}$ of each primer (Table 1 ), $2 \mathrm{mM}$ of each dNTP (FINZYMES), $1 \mu \mathrm{l}(100 \mathrm{ng})$ of genomic DNA extract, $1 \mathrm{U}$ of DNA polymerase (FINZYMES), and $5 \mu \mathrm{l}$ of supplied PCR buffer. Amplification was done using GeneAmp PCR system 2400 (PERKIN ELMER) using the following profile: initial denaturation at $95^{\circ} \mathrm{C}$ for 5 minutes, 30 cycles of denaturation at $95^{\circ} \mathrm{C}$ for 1 minute, annealing at $55^{\circ} \mathrm{C}$ for 1 minute, and extension at $72^{\circ} \mathrm{C}$ for 1 minute/1000 nucleotides, and a final extension at $72^{\circ} \mathrm{C}$ for 7 minutes. Amplified products were detected by agarose gel electrophoresis in 1\% TrisAcetate-EDTA (TAE) agarose (Research organics, inc. USA) stained with ethedium bromide.

Hybridization for intI1 detection and bla $_{\mathrm{VEB}-1}$ localization: Class 1 integron integrase gene (intI1) and $b l a_{\mathrm{VEB}-1}$ was amplified from the plasmid pCTF202 using the primer sets, IntI1F/IntI1R and VEB1F/VEB1R, respectively. The $471 \mathrm{bp}$ and $642 \mathrm{bp}$ amplicons generated were purified using the PCR product purification kit (PCR clean up kit). Both of these DNA were labeled with the random primer labeling kit (Roche, USA). Dot blot and southern blot hybridization were performed on these chromosomal DNA as per the manufacturer's protocol (Amersham, UK). EcoRI restriction enzyme was used for southern blot hybridization. Plasmids pCTF202 and pUC19 were used as positive and negative controls, respectively. Ethical clearance was taken from the institution where the study was conducted.

\section{RESULTS}

\section{Detection of IntI1 and resistant cassettes}

The dot blot hybridization using $471 \mathrm{bp}$ amplicon of intI1 gene as a probe was able to detect intI1in 30 isolates $(81.08 \%)$. The dot blot negative isolates were Ab08-5, -6, -07, -24, -25, -26, and -28 (Fig.1). 
Table 1. Primers used in this study

\begin{tabular}{|c|c|c|c|}
\hline Primer Name & Target & Amplicon (bp) & Primer sequence $\left(5^{\prime}-3^{\prime}\right)$ \\
\hline IntI1F & IntI1 & 471 & AAGGATCGGGCCTTGATGTT \\
\hline IntI1R & & & CAGCGCATCAAGCGGTGAGC \\
\hline 5 'CS & Variable region of class 1 & variable & GGCATCCAAGCAGCAAG \\
\hline 3'CS & integron element & varıabie & AAGCAGACTTGACCTGA \\
\hline VEB1F & $b B l a_{\mathrm{VEB}-1}$ & 642 & CCACTTCCATTTCCCGATGC \\
\hline VEB1R & & & GGACTCTGCAACAAATACGC \\
\hline CMLAF2 & $\mathrm{cmlA}$ & 455 & ACTAATGATGGCAGGCAAG \\
\hline CMLAR2 & & & AAGACAGACCGAGCACGACT \\
\hline ARR-2F & arr-2 & 417 & CATTTCGAGGACGGTCGTAT \\
\hline ARR2-R & & & GCCTATTGCGCATAAAATGG \\
\hline OXA-10F & bla 0 XX-10 & 261 & TTAGGCCTCGCCGAAGCG \\
\hline OXA-10R & & & CTTTGTTTTAGCCACCACCAATGATG \\
\hline
\end{tabular}

$\mathrm{F}$, forward; $\mathrm{R}$, reverse primer

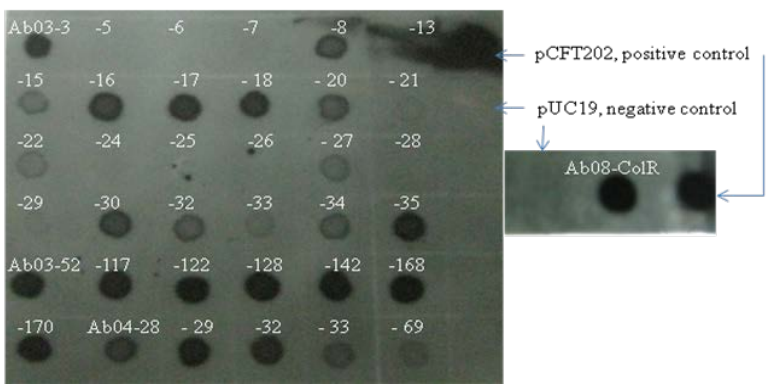

Fig 1: Dot blot hybridization using the intI1 probe for the detection of intI1 gene. Thirty isolates were positive for intI1 gene. pCFT202 carrying class 1 integron element was used as a positive control and plasmid pUC19 was used as negative control.

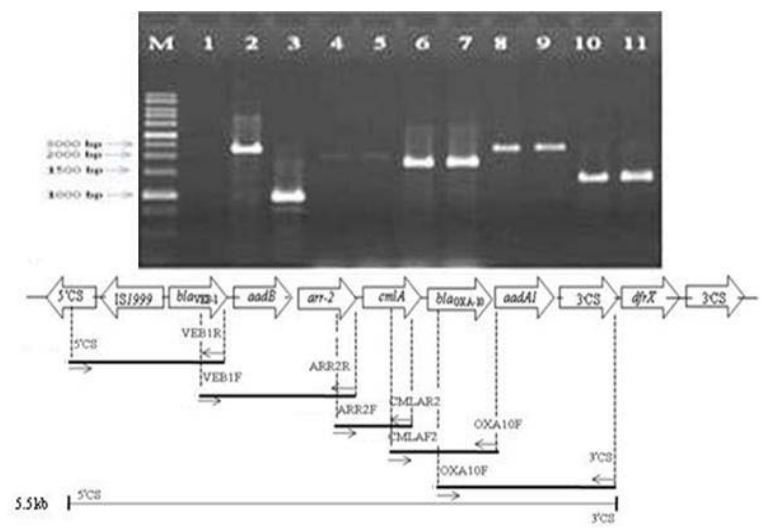

Figure 2: PCR mapping of $b l a_{\mathrm{VEB}-1}$ containing class 1 integron element from isolate Ab03-168. Lane $\mathrm{M}=$ Molecular weight marker $(1 \mathrm{~Kb}+$, invitrogen), Lane $1=$ Negative control, Lanes $2,4,6,8$, and 10 are positive control for fragments $5^{\prime} \mathrm{CS}-b l a_{\mathrm{VEB}-1}$, $b l a_{\text {VEB-1- }}-a r r-2, a r r-2-c m l A, \quad c m l A-b l a_{\text {OXA-10 }}$, and $b l a_{\text {OXA }-10}-3$ 'CS from Lane $3=5^{\prime}$ 'CS- bla $a_{\mathrm{VEB}-1}$ from $P$. aeruginosa strain 58. Lanes $3,5,9$, and 11 represents fragments $b l a_{\text {VEB-1-arr-2, }}$ arr-2-cmlA, cmlA-bla $a_{\mathrm{OXA}-10}$, and $b l a_{\mathrm{OXA}-10}-3^{\prime} \mathrm{CS}$ from isolate $\mathrm{Ab} 03-$ 168).

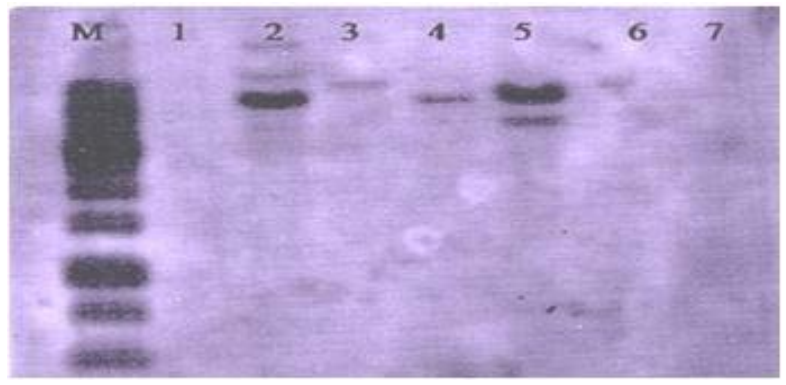

Figure 3 Genetic localization of bla $a_{\mathrm{VEB}-1}$ gene in $b l a_{\mathrm{VEB}-1}$ gene positive isolates. Signals were detected only in four lanes. The fragments generated using EcoRI was different in different isolates suggesting different location of bla $a_{\mathrm{VEB}-1}$. Lane $\mathrm{M}=$ Molecular weight marker (1 $\mathrm{kb}^{+}$, SibZymes), Lane 2-5 represents isolates; Ab03-52, Ab03-117, Ab03-122, and Ab03-168, respectively.

Moreover, two isolates (Ab08-25 and -26) were also intI1positive by intI1 PCR. Altogether, 32 isolates were positive for class 1 integron element (86.48\%). PCR amplification detected bla $a_{\mathrm{VEB}-1,}$, bla $a_{\text {OXA-10, }}$ arr-2, and $\mathrm{cmlA}$ in 7 (Ab08-15, Ab03-52, -117, -122, -168, Ab04-29, Ab04-32), 10 (Ab08-20, -25, -26, -27, -29, Ab03-52, -117, -122, -168, Ab04-29), 5 (Ab08-15, Ab03-117, -168, Ab04-29, -32), and 9 (Ab08-15, -16, $-21, \mathrm{Ab} 03-52,-117,-122,-168$, Ab04-29, -32) isolates, respectively from intI1 positive isolates.

\section{Amplification of variable region of class 1 integron element}

The variable region of class 1 integron element was amplified from 32 intI1 positive isolates using the consensus primer set $5^{\prime} \mathrm{CS}$ and $3^{\prime} \mathrm{CS}$. Numerous variable regions ranging from 0 to 5 were amplified (data not shown). Smallest variable region amplified was $0.5 \mathrm{~kb}(\mathrm{n}=16)$ and the largest was 5.5 $\mathrm{kb}(\mathrm{n}=4)$. 
Commonest variable region that was amplified was $0.7 \mathrm{~kb}(\mathrm{n}=23)$. Variable region sized $5.5 \mathrm{~Kb}$ was amplified from 4 isolates (Ab03-168, Ab04-28, Ab08-20, and Ab08-22) and only isolate Ab03-168 was positive for $b l a_{\mathrm{VEB}-1}$.

\section{Characterization of $\boldsymbol{b l a}_{\mathrm{VEB}-1}$ containing integron element}

The only class 1 integron element carrying the bla $a_{\text {VEB-1 }}$ gene with a variable region of $5.5 \mathrm{~Kb}$ in an isolate Ab03-168 was characterized by PCR mapping (5'CS-VEB1R, VEB1F-ARR 2R, ARR 2F-CMLA R2, CMLA F2-OXA-10R, and OXA-10R-3'CS). The gel electrophoresis of amplicons detected is shown in Fig 2. The class 1 integron element revealed was 5'CS-aadB-bla $a_{\text {VEB-1- }}$ arr-2-cmlA-aadA1-bla ${ }_{\text {OXA-10-3'CS. }}$

\section{$b l a_{\text {VEB-1 }}$ localization}

Southern blot was performed with bla $a_{\text {VEB-1 }}$ specific probe to interrogate the genetic localization of $b l a_{\text {VEB-1 }}$ in $b l a_{\text {VEB-1 }}$ positive isolates $(\mathrm{n}=7)$. The signal was detected for 4 isolates (Fig 3). The signals generated were different in different isolates, two to three bands (Ab03-52, Ab03-168) and a single band (Ab03-117, Ab03-122). The genetic environment surrounding either at upstream or downstream of $b a_{\mathrm{VEB}-1}$ gene was different in different isolates.

\section{DISCUSSION}

The class 1 Integron element harbors multiple resistance cassettes and confer resistance to numerous antibiotics. This is a hallmark of resistance phenotype in $A$. baumannii. ${ }^{1}$ The existence of this mobile genetic element was sought by dot blot hybridization and intI1 PCR. The prevalence of class 1 integron element in our isolates was $86.48 \%$. High prevalence of class 1 integron element have also been reported elsewhere. ${ }^{12,13}$ Multidrug resistance pattern of these isolates might have correlated with the presence of this integron element. ${ }^{11}$

The largest class 1 integron element in GRI, AbaR1 is $5.5 \mathrm{~kb}$ which is present in European clone I, AYE and harbors multiple resistant cassettes (bla $a_{\mathrm{VEB}-1}$, aadB, arr-2, cmlA bla $0 \times \mathrm{XA}-10$, and aadA1). ${ }^{1}$ The resistant cassettes (bla $a_{\mathrm{VEB}-1}, a r r-2, c \mathrm{cmlA}$ and bla $\left.\mathrm{OXA}_{\mathrm{X}-10}\right)$ and variable regions of class 1 integron element from these isolates were amplified. The resistant cassettes; bla $a_{\text {VEB-1 }} \quad(\mathrm{n}=7), \quad$ bla $a_{\text {OX-10 }} \quad(\mathrm{n}=9), \quad$ arr-2 $(\mathrm{n}=10)$, and $\mathrm{cmlA}(\mathrm{n}=5)$ were detected. All of these isolates were also positive for intI1. The variable region of $5.5 \mathrm{~kb}$ was amplified in 4 isolates (Ab08-
20, -22, Ab04-168, and Ab04-28) while bla $a_{\mathrm{VEB}-1}$ gene was only present in an isolate, Ab08-168. The integron from this isolate was characterized by PCR mapping. The cassette found was 5 'CS-intI1-bla $a_{\text {VEB-1- }}$ aadB-arr-2-aadA1-cmlA-bla 0 XX-10-3'CS. Gene cassettes, $a a d B$ and $a a d A 1$ were not amplified in this study however, bla $a_{\text {VEB-1-arr-2 (2000 bp) and bla }}$ OXA${ }_{10}-3^{\prime} \mathrm{CS}(1500 \mathrm{bp})$ amplicons were of accurate size to accommodate for $a a d B$ and aadA1, respectively.

The common structure of class 1 integron element that is widely disseminated among Enterobacteriaceae is 5'CS-IS1999-bla ${ }_{\mathrm{VEB}-1}$-aadB-arr-

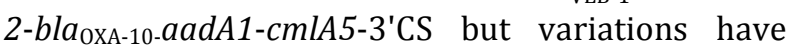
been reported. ${ }^{1}$ The cassette organization that was found in Ab03-168 was similar to European clone I, AYE, except IS1999 was missing between 5'CS and bla $_{\text {VEB-1 }}$ (Figure 2). Tribuddharat et al. described IS1999 element primarily in $P$. aeruginosa from Thailand and was integrated upstream of $b a_{\text {VEB- } 1}$ gene cassette. ${ }^{9}$ This insertion sequence provides a strong promoter for the expression of this gene. ${ }^{14}$ Since, isolate Ab03-168 possess carbapenemase OXA-23 and can hydrolyze extended spectrum cephalosporins (ESCs), IS1999 might not be required for high level expression of $b l a_{\mathrm{VEB}-1} \cdot{ }^{1}$ Furthermore, carbapenems are the first choice of treatment for this superbug so the withdrawal of ESCs might have helped IS1999 to shuffle around the genome. The integron structure without IS1999 was seen in $P$. aeruginosa from France. ${ }^{15}$ These variations were further supported by our finding that other isolates; Ab08-15, Ab03-52, -117, -122, Ab04-29, and -32 were also positive for bla VEB-1 $_{1}$ but, neither of these isolates was positive for integron element of size 5.5 $\mathrm{kb}$. The bla $a_{\text {VEB-1 }}$ was localized by southern blot in isolates carrying bla $a_{\mathrm{VEB}-1}$ and the genetic environment of bla $a_{\mathrm{VEB}-1}$ was different in different isolate. This also suggests that the genetic make up of isolates carrying the $b l a_{\mathrm{VEB}-1}$ gene was different and hence different integron structure.

The integron element with slight variation was widely disseminated in other Gram-negative bacteria like; P. aeruginosa, E. coli, E. cloacae, and $K$. pneumoniae in this hospital and abroad.,10 This suggests the interspecies horizontal gene transfer and international dissemination of this mobile genetic element among international clones of Enterobacteriaceae and minor variation brought about by selective antibiotic pressure in different geographical areas. ${ }^{16}$

\section{CONCLUSIONS}

$b l a_{\mathrm{VEB}-1}$ containing class 1 integron element with 
cassette organization similar to the one present in $A$. baumannii, European clone I, AYE was identified in Thai $A$. baumannii. Presence of common integron element in international clones of $A$. baumannii isolates implies the mating of our clones with international clones at least once or spread of our clones internationally. Monitoring and surveillance of the global spread of multi-resistant $A$. baumannii is mandatory to control the spread of resistant gene cassettes and this multi-resistant pathogen.

CONFLICT OF INTEREST: None to declare

FINANCIAL INTEREST: None to declare

\section{REFERENCES}

1. Fournier PE, Vallenet D, Barbe V, et al. Comparative genomics of multidrug resistance in Acinetobacter baumannii. PLoS Genet 2006; 2: 62-72.

2. Michele I, Laura V, Daniela F, et al. Whole-Genome pyrosequencing of an epidemic multidrug-resistant Acinetobacter baumannii strain belonging to the European Clone II group. Antimicrob Agents Chemother 2008; 25: 2616-25.

3. Adams MD, Goglin K, Molyneaux N, et al. Comparative Genome Sequence Analysis of Multidrug-Resistant Acinetobacter baumannii. J Bacteriol 2008; 190: 8053-64.

4. Peleg AY, Seifert H, Paterson DL. Acinetobacter baumannii: Emergence of a successful pathogen. Clin Micobiol Rev 2008; 21: 538-82.

5. Stokes HW, Hall RM. A novel family of potentially mobile DNA elements encoding site-specific geneintegration functions: integrons. Mol Microbiol 1989; 3: 1669-83.

6. Wroblewska MM, Towner KJ, Marchel H, Luczak M. Emergence and spread of carbapenem-resistant strains of Acinetobacter baumannii in a tertiary-care hospital in Poland. Clin Microbiol Infect 2007; 13: 490-96.

7. Navia MM, Ruiz J, Vila J. Characterization of an integron a new class $\mathrm{D} \beta$-Lactamase (OXA-37) in Acinetobacter baumannii. Microbial Drug Res 2008; 2: 261-65.

8. Tribuddharat C. Mechanisms of antibiotic resistance in Pseudomonas aeruginosa. North Chicago: Finch University of Health Sciences/Medical School (Now known as Rosalind Franklin University of Medicine and Science); 1999.

9. Tribuddharat C, Fennewald M. Integron mediated rifampin resistance in Pseudomonas aeruginosa. Antimicrob Agents Chemother 1999; 43: 960-62.

10. Girlich D, Poirel L, Leelaporn A, et al. Molecular epidemiology of the integron-located VEB-1 extendedspectrum $\beta$-lactamase in Nosocomial Enterobacterial Isolates in Bangkok, Thailand. J Clin Microbiol 2001; 39: 175-82.

Thapa B, Tribuddharat C, Srifuengfung S, Dhiraputra C. High prevalence of bla $a_{0 \times-23}$ in oligoclonal carbapenemresistant Acinetobacter baumannii in Siriraj Hospital, Mahidol University, Bangkok, Thailand. Southeast Asian J
Trop Med Public Health 2010; 41:625-35.

12. Segal H, Thomas R, Elisha BG. Characterization of class 1 integron resistance gene cassettes and the identification of novel IS-like element in Acinetobacter baumannii. Plasmid 2003; 2:169-178.

13. Ploy MC, Denis F, Courvalin P, Lambert T. Molecular Characterization of Integrons inAcinetobacter baumannii: Description of a Hybrid Class 2 Integron. Antimicro Agents Chemother 2000:10: 2684-88.

14. Aubert D, Naas T, Nordmann P. IS1999 increases expression of the extended-spectrum $\beta$-lactamase VEB-1 in Pseudomonas aeruginosa. J Bacteriol 2003; 185: 531419.

15. Mugnier P, Dubrous P, Casin I, Arlet G, Collatz E. A TEM-derived extended-spectrum b-lactamase in Pseudomonas aeruginosa. Antimicrob Agents Chemother 1996; 40:2488-93.

16. Girlich D, Naas T, Leelaporn A, Poirel L, Fennewald M, P Nordmann. Nosocomial Spread of the integron-located veb-1-like Cassette Encoding an extended-spectrum $\beta$ Lactamase in Pseudomonas aeruginosa in Thailand. Clin Infect Dis 2002; 34: 603-11.

\section{Citing this article}

Thapa B, Tribuddharat C, Srifuengfung S, Dhirapurta C. Class 1 integron element in Thai Acinetobacter baumannii reveals a linkange to European clone I. Int J Infect Microbiol 2012;1(1):24-28. 